\title{
Elevated homocysteine levels and risk of cardiovascular and all-cause mortality: a meta-analysis of prospective studies
}

\author{
Hui-yong PENG, Chang-feng MAN, Juan XU, Yu FAN ${ }^{\dagger *}$ \\ (Cancer Institute, the Affiliated People's Hospital, Jiangsu University, Zhenjiang 212002, China) \\ †E-mail: jszjfanyu@163.com \\ Received June 30, 2014; Revision accepted Nov. 6, 2014; Crosschecked Dec. 20, 2014
}

\begin{abstract}
Objective: To investigate whether elevated homocysteine levels were a predictor of subsequent coronary heart disease (CHD) mortality, cardiovascular mortality or all-cause mortality in the general population by a metaanalysis. Methods: In a systematic search conducted in the databases of PubMed and Embase prior to October 2013, we identified relevant prospective observational studies evaluating the association between baseline homocysteine levels and CHD mortality, cardiovascular or all-cause mortality in the general population. Pooled adjust risk ratio (RR) and corresponding 95\% confidence interval $(\mathrm{Cl})$ were calculated separately for categorical risk estimates and continuous risk estimates. Results: Twelve studies with 23623 subjects were included in the meta-analysis. Comparing the highest to lowest homocysteine level categories, CHD mortality increased by $66 \%(\mathrm{RR} 1.66 ; 95 \% \mathrm{Cl} 1.12-2.47$; $P=0.012$ ), cardiovascular mortality increased by $68 \%$ (RR 1.68; 95\% Cl 1.04-2.70; $P=0.033$ ), and all-cause mortality increased by $93 \%$ (RR 1.93; 95\% Cl 1.54-2.43; $P<0.001$ ). Moreover, for each $5 \mu \mathrm{mol} / \mathrm{L}$ homocysteine increment, the pooled RR was $1.52(95 \% \mathrm{Cl} 1.26-1.84 ; P<0.001)$ for CHD mortality, $1.32(95 \% \mathrm{Cl} 1.08-1.61 ; P=0.006)$ for cardiovascular mortality, and $1.27(95 \% \mathrm{Cl} 1.03-1.55 ; P=0.023)$ for all-cause mortality. Conclusions: Elevated homocysteine levels are an independent predictor for subsequent cardiovascular mortality or all-cause mortality, and the risks were more pronounced among elderly persons.
\end{abstract}

Key words: Homocysteine, Coronary heart disease, Cardiovascular mortality, All-cause mortality, Meta-analysis doi:10.1631/jzus.B1400183 Document code: A CLC number: R446

\section{Introduction}

Coronary heart disease (CHD) and cardiovascular mortality represent significant health and social problems in the world. The commonly recognized risk factors, including hyperlipidemia, hypertension, obesity, diabetes, and smoking, have been identified as mortality risk assessments in the general population. However, these characteristics do not fully explain all the mortality risks. Early detection of new risk factors would be beneficial for predicting death and reducing mortality.

\footnotetext{
${ }^{\ddagger}$ Corresponding author

(1D) ORCID: Yu FAN, http://orcid.org/0000-0003-4095-8427

(C) Zhejiang University and Springer-Verlag Berlin Heidelberg 2015
}

Homocysteine (Hcy) is a sulfur-containing amino acid produced during catabolism of the essential amino acid methionine. Elevated serum Hcy levels have been implicated with the risks of CHD and cardiovascular disease (CVD). The prevalence estimates of hyperhomocysteinemia $(>14 \mathrm{mmol} / \mathrm{L})$ vary between $5 \%$ and $30 \%$ in the general population (Selhub et al., 1993; Nygård et al., 1995). Some epidemiologic studies (Hoogeveen et al., 2000; Vollset et al., 2001; Blacher et al., 2002; Gonzalez et al., 2007; Cui et al., 2008; Dangour et al., 2008; Waśkiewicz et al., 2012; Wong et al., 2012) but not all (Stehouwer et al., 1998; Kark et al., 1999; de Bree et al., 2003; Swart et al., 2012) indicated that the elevated Hcy represents a risk factor for total death, CHD death, or cardiovascular mortality in the general 
population; additionally, this incremental increase in risk was pronounced in high risk subjects (Nygård et al., 1997; Zylberstein et al., 2004; Rossi et al., 2006; Naess et al., 2013). Furthermore, two conducted studies (Bostom et al., 1999; Colon Lopez et al., 2008) were difficult to compare because the Hcy cut-off values varied. Therefore, the associations of elevated Hcy levels and mortality risk remained conflicting.

Given that the results of these studies have not yet been quantitatively evaluated, a meta-analysis would help to classify this issue. Here, we present a meta-analysis of prospective studies to determine the magnitude of Hcy levels and CHD, cardiovascular and all-cause mortality in the general population.

\section{Materials and methods}

\subsection{Search strategy}

The meta-analysis was conducted according to the checklist of the meta-analysis of observational studies in epidemiology (Stroup et al., 2000). The literature search was conducted in PubMed and Embase databases published prior to October 2013. Potentially relevant studies included the following keywords: mortality/death, coronary heart disease death/mortality, and cardiovascular death/mortality, in combination with hyperhomocysteinemia or homocysteine, prospective, and follow-up. Moreover, the reference lists of the relevant publications were manually searched to identify additional studies.

\subsection{Study selection}

Studies included in the meta-analysis should meet the following criteria: (1) prospective observational study was conducted in the general population; (2) the exposure was plasma or serum Hcy; (3) the outcome was all-cause mortality or CHD and cardiovascular mortality; (4) provided at least the age-adjusted risk ratio (RR), hazard ratio (HR), or odds ratio (OR) with their confidence intervals (CIs) for categorical risk estimates and continuous risk estimates. The outcome measures included CHD death, cardiovascular death (defined as death from myocardial infarction, heart failure, stroke or arrhythmia), and all-cause mortality. Mortality at the end of follow-up was obtained from the official death certificates, medical records, or personal interviews.
Studies were excluded if they met the following criteria: (1) subjects were in a highly selected disease group; (2) the design was a retrospective study, review, and/or case report; (3) only provided a risk estimate with no means by which to calculate the $95 \%$ CI or unadjusted OR or RR was reported.

\subsection{Data extraction and quality assessment}

From eligible studies, two authors (Peng H.Y. and $\mathrm{Xu} \mathrm{J.)} \mathrm{independently} \mathrm{extracted} \mathrm{the} \mathrm{data.} \mathrm{Any}$ disagreement was settled by discussion. We did not contact authors to request additional information when data were not available. The following items were extracted from the included papers: first author, year of publication, study design, country, sample size, gender, age, Hcy comparison, death assessment, mortality events, RR or OR with its $95 \% \mathrm{CI}$, duration of follow-up, and statistical adjustments for confounding factors.

The quality of all the included studies was assessed by the following aspects according to the Newcastle-Ottawa scale (NOS) (Wells et al., 2013): selection of study groups, comparability of the study groups, and reporting of important outcomes. Using the scale, we assigned a number of stars based on the answer options of each study. A maximum of two stars was assigned for comparability. The total NOS star count ranges $0-9$. A score of $\leq 5$ indicated a high risk of bias.

\subsection{Statistical analysis}

All statistical analysis was performed using the STATA statistical software (Version 12.0, Stata Corp LP, College Station). $P<0.05$ was considered statistically significant. Since nested case-control and cohort studies are all prospective studies, for the purpose of meta-analysis, ORs, and HRs were considered as approximations of RRs. We used the highest versus the lowest category as the measure of the relationships between Hcy levels and risk estimates. In addition, we also pooled risk estimates for per $5 \mu \mathrm{mol} / \mathrm{L}$ increment serum Hcy levels. Homogeneity among studies was determined using the Cochrane Q statistic $(P<0.10$ was considered indicative of statistically significant heterogeneity) and $I^{2}$ statistic (values of more than $50 \%$ as significant heterogeneity) (Higgins et al., 2003). When there was no evidence of significant heterogeneity, effect sizes were determined 
using a fixed-effects model, otherwise we used a random effects model. Begg's rank correlation test (Begg and Mazumdar, 1994) and Egger's linear regression test (Egger et al., 1997) at $P<0.10$ were employed to assess potential publication bias. A sensitivity analysis was carried out by sequentially omitting individual studies at each turn with the metaninf command in STATA.

\section{Results}

\subsection{Literature search}

The detailed steps of the study selection are presented in Fig. 1. Through the initial literature search, a total of 960 potentially relevant publications were identified. After screening the abstracts or titles and reviewing the full texts, 12 studies (Stehouwer $e t$ al., 1998; Kark et al., 1999; Hoogeveen et al., 2000; Vollset et al., 2001; Blacher et al., 2002; de Bree et al., 2003; Gonzalez et al., 2007; Cui et al., 2008; Dangour et al., 2008; Swart et al., 2012; Waśkiewicz et al., 2012; Wong et al., 2012) met our inclusion criteria.

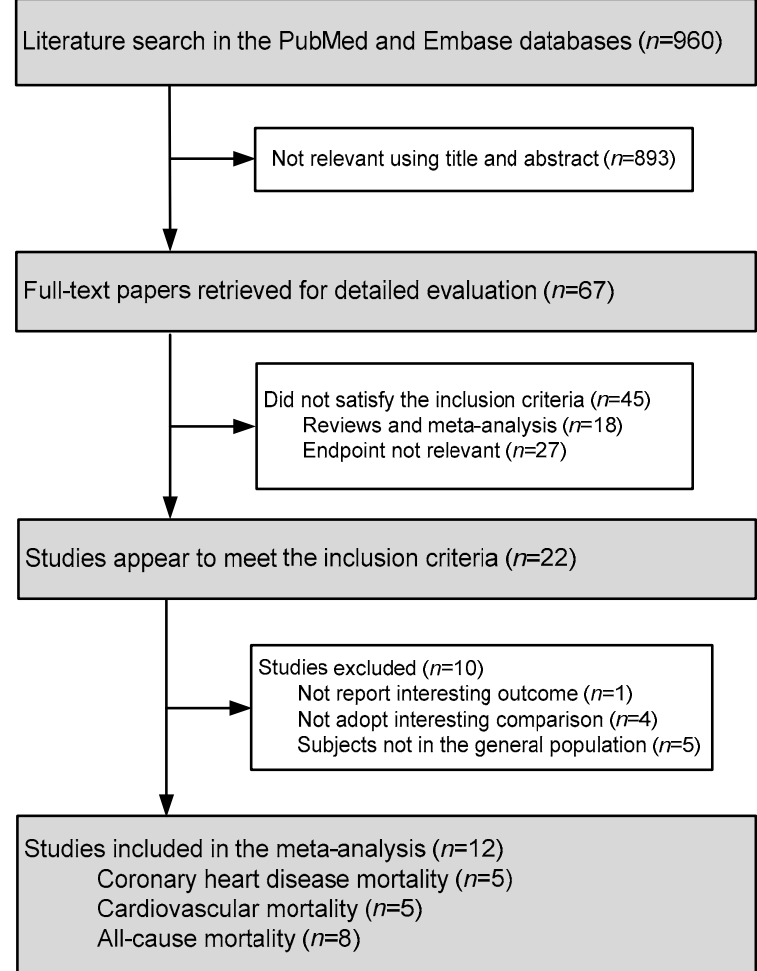

Fig. 1 Flow chart of study selection process for meta-analysis

\subsection{Study characteristics and quality assessment}

The characteristics of the papers in the metaanalysis are listed in Table 1. All studies were published between 1998 and 2012. In total, the studies included 23623 participants. Most participants were represented from the elderly population except for two studies (de Bree et al., 2003; Waśkiewicz et al., 2012), which included a wide range population. The follow-up duration across studies ranged from 4.1 to 14 years. The sample size ranged from 264 to 7165 . Most of the studies were conducted in predominantly European countries. The NOS for assessing the quality of the included studies are listed in Table 2, and the scores ranged from 5 to 7 .

\subsection{All-cause mortality}

Six studies (Kark et al., 1999; Vollset et al., 2001; Gonzalez et al., 2007; Dangour et al., 2008; Swart et al., 2012; Waśkiewicz et al., 2012) reported an outcome on all-cause mortality on the highest versus lowest Hcy categories. The total number of participants included in this meta-analysis was 15904 with 1936 death events. As shown in the part 1 of Fig. 2, there was a statistically significant heterogeneity among the studies $\left(I^{2}=52.3 \%, P=0.05\right)$, and the random effects model was used to pool the results. Overall, elevated serum Hcy levels were associated with a greater risk of all-cause mortality (RR 1.93; 95\% CI 1.54-2.43; $P<0.001)$. Publication bias was not indicated in Begg's rank correlation test $(P=0.368)$ or Egger's linear regression test $(P=0.937)$. As shown in Fig. 3, in the subgroup analysis based on the gender of the two studies (Kark et al., 1999; Swart et al., 2012), women with elevated Hcy levels increased the risk of all-cause mortality (RR 1.74; 95\% CI 1.242.44; $P=0.001)$; in contrast, no statistical significance was found for the men (RR1.87; 95\% CI 0.64-5.50; $P=0.255$ ).

A significant dose-response association, between serum Hcy levels and the risk of all-cause mortality, was noted in three studies (Hoogeveen et al., 2000; Vollset et al., 2001; Wong et al., 2012). The total number of participants included in this metaanalysis was 9825 , with 1179 death events. As shown in the part 2 of Fig. 2, there was a statistically significant heterogeneity among the studies $\left(I^{2}=86.8 \%\right.$, $P=0.001$ ), the random effects model was used to pool the results. The pooled RR of all-cause mortality for the per $5 \mu \mathrm{mol} / \mathrm{L}$ increment serum Hcy was 1.27 (95\% CI 1.03-1.33; $P=0.023)$. 


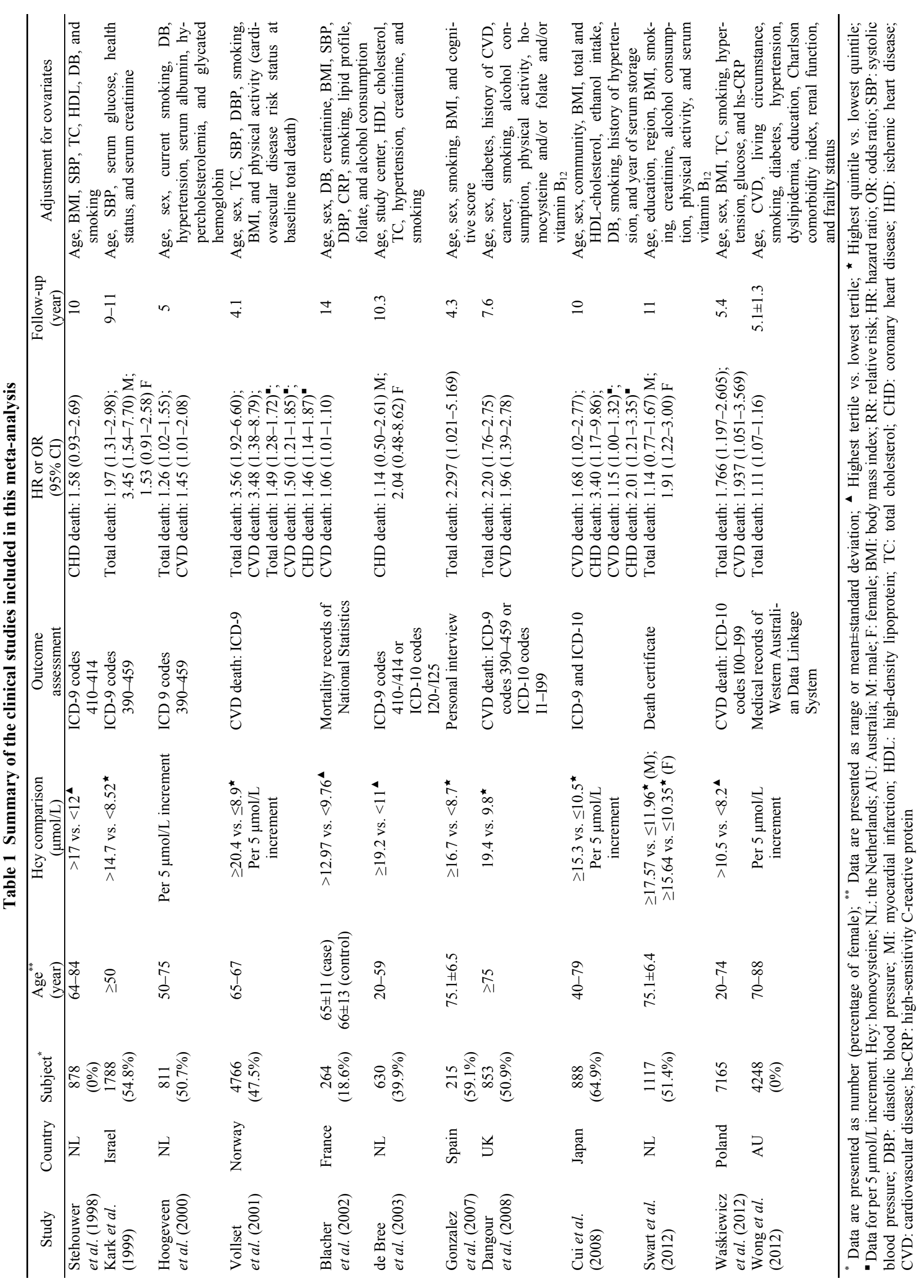


Table 2 Quality assessment of studies included in meta-analysis

\begin{tabular}{lccccccccc}
\hline \multicolumn{1}{c}{ Study } & A1 & A2 & A3 & A4 & A5 & A6 & A7 & A8 & Overall score \\
\hline Stehouwer et al. $(1998)$ & $*$ & $*$ & $*$ & $*$ & $*$ & $*$ & $*$ & & 7 \\
Kark et al. $(1999)$ & $*$ & $*$ & $*$ & $*$ & $*$ & $*$ & $*$ & $*$ & 8 \\
Hoogeveen et al. $(2000)$ & $*$ & $*$ & $*$ & $*$ & $*$ & $*$ & $*$ & & 7 \\
Vollset et al. $(2001)$ & $*$ & $*$ & $*$ & & $*$ & $*$ & & $*$ & 6 \\
Blacher et al. $(2002)$ & $*$ & & $*$ & $*$ & $*$ & $*$ & $*$ & & 6 \\
de Bree et al. (2003) & $*$ & $*$ & $*$ & $*$ & $*$ & $*$ & $*$ & $*$ & 8 \\
Gonzalez et al. $(2007)$ & $*$ & $*$ & $*$ & $*$ & $*$ & & & & 5 \\
Dangour et al. $(2008)$ & $*$ & $*$ & $*$ & & $* *$ & $*$ & $*$ & $*$ & 8 \\
Cui et al. $(2008)$ & $*$ & & $*$ & $*$ & $*$ & $*$ & $*$ & & 6 \\
Swart et al. $(2012)$ & $*$ & $*$ & $*$ & $*$ & $* *$ & $*$ & $*$ & $*$ & 9 \\
Waśkiewicz et al. $(2012)$ & $*$ & $*$ & $*$ & $*$ & $*$ & $*$ & $*$ & & 8 \\
Wong et al. $(2012)$ & $*$ & $*$ & $*$ & & $*$ & $*$ & & & 5
\end{tabular}

\# Using the Newcastle-Ottawa scale (NOS) (Wells et al., 2013), the number of stars based on the answer options of each study was assigned. A maximum of two stars was assigned for comparability. The total NOS star count ranges $0-9$. A score of $\leq 5$ indicated a high risk of bias. A1: representativeness of the exposed cohort; A2: selection of non-exposed cohort; A3: ascertainment of exposure; A4: demonstration that outcome was not present at study start; A5: comparability of cohorts on the basis of the design or analysis; A6: assessment of outcome; A7: enough follow-up periods; A8: lost to follow-up acceptable (reported $<10 \%$ )

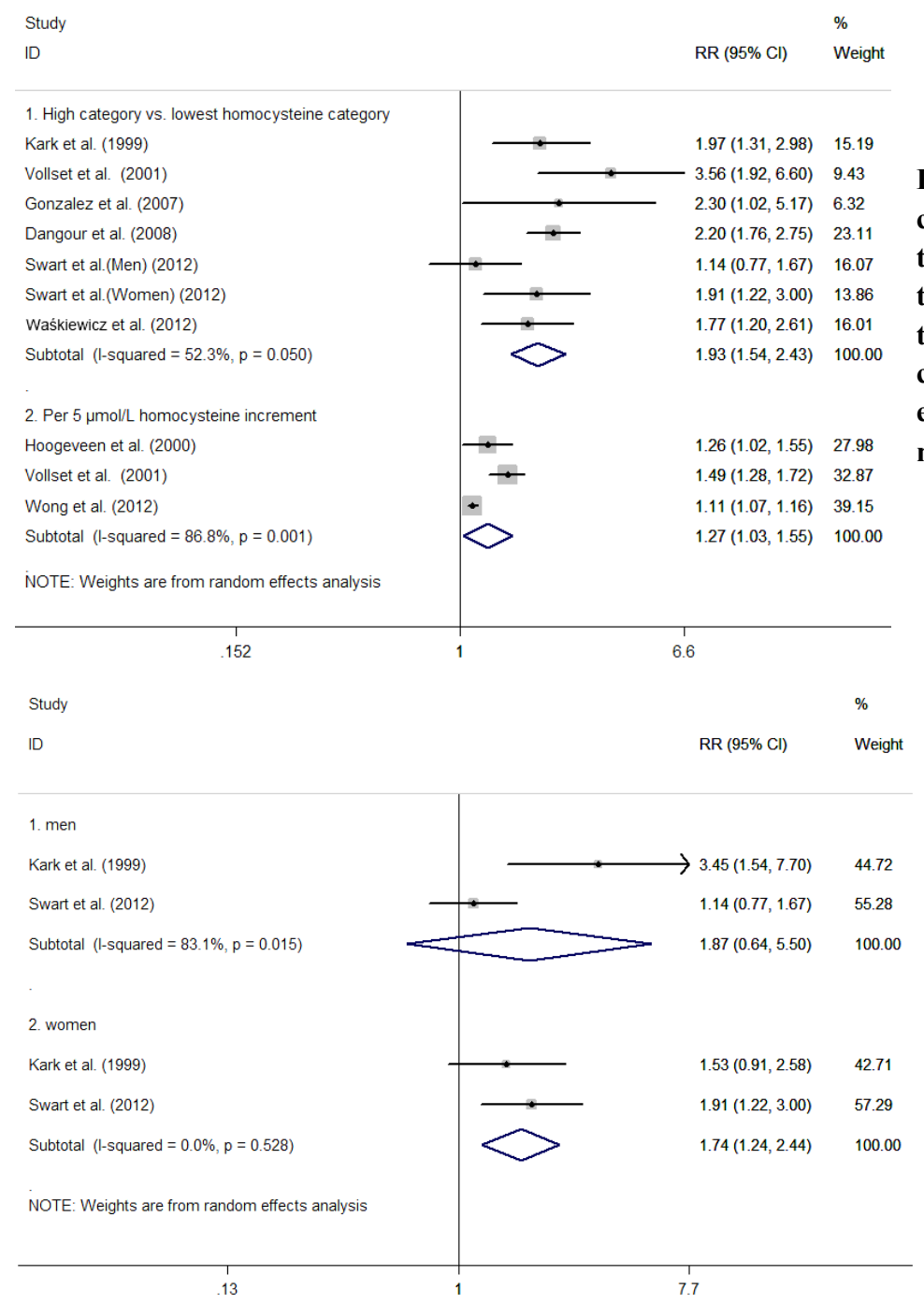

Fig. 2 Risk ratio (RR) and 95\% confidence interval (CI) from the eligible studies of homocysteine levels and all-cause mortality calculated separately for categorical and continuous risk estimates in a random effects model

Fig. 3 Risk ratio (RR) and 95\% confidence interval (CI) of homocysteine levels and all-cause mortality calculated separately for gender in a random effects model 


\subsection{Cardiovascular mortality}

Four studies (Vollset et al., 2001; Blacher et al., 2002; Cui et al., 2008; Dangour et al., 2008) reported outcomes on cardiovascular mortality on the highest versus lowest Hcy levels categories. The total number of participants, included in this meta-analysis, was 6771 with 860 cardiovascular death events. As shown in the part 1 of Fig. 4, there was a statistically significant heterogeneity among the studies $\left(I^{2}=85.9 \%\right.$, $P=0.000)$, the random effects model was used to pool the results. Overall, elevated serum Hcy levels were significantly associated with an increased cardiovascular mortality risk (RR 1.68; 95\% CI 1.04-2.70; $P=0.033)$. Publication bias was observed in Begg's rank correlation test $(P=0.734)$, but not in the Egger's linear regression test $(P=0.039)$.

A significant dose-response association between the serum Hcy levels and risk of cardiovascular mortality was indicated in the three studies (Hoogeveen et al., 2000; Vollset et al., 2001; Cui et al., 2008). The total number of participants included in this metaanalysis was 6465, with 641 cardiovascular death events. As shown in the part 2 of Fig. 4, there was a statistically significant heterogeneity among the studies $\left(I^{2}=58.6 \%, P=0.089\right)$, the random effects model was used to pool the results. The pooled RR of cardiovascular mortality for the per $5 \mu \mathrm{mol} / \mathrm{L}$ increment serum Hcy level was 1.32 (95\% CI 1.08-1.61; $P=0.006)$.

\subsection{CHD mortality}

Three studies (Stehouwer et al., 1998; de Bree et al., 2003; Cui et al., 2008) reported the outcome on CHD mortality in the highest versus lowest Hcy level categories. The total number of participants included in this meta-analysis was 2396 with 334 CHD death events. As shown in the part 1 of Fig. 5, there was no significant heterogeneity among the studies $\left(I^{2}=0 \%\right.$, $P=0.45)$, the fixed-effects model was used to pool the results. Overall, elevated serum Hcy levels were significantly associated with an increased CHD mortality risk (RR 1.66; 95\% CI 1.12-2.47; $P=0.012$ ). However, after we excluded one study (Cui et al., 2008), the sensitivity analysis only showed some trend, this positive association was not statistically significant (RR 1.48; 95\% CI 0.97-2.27; $P=0.071$ ).

A significant dose-response association between serum Hcy levels and the risk of CHD mortality was indicated in three studies (Hoogeveen et al., 2000; Vollset et al., 2001; Cui et al., 2008). The total number of participants included in this meta-analysis was 6207, with 344 CHD death events. As shown in the part 2 of Fig. 5, there was no significant heterogeneity among the studies $\left(I^{2}=0 \%, P=0.516\right)$, the fixed-effects model was used to pool the results. The pooled RR of CHD mortality for per $5 \mu \mathrm{mol} / \mathrm{L}$ increment Hcy was $1.52 \quad(95 \%$ CI 1.26-1.84; $P<0.001)$.

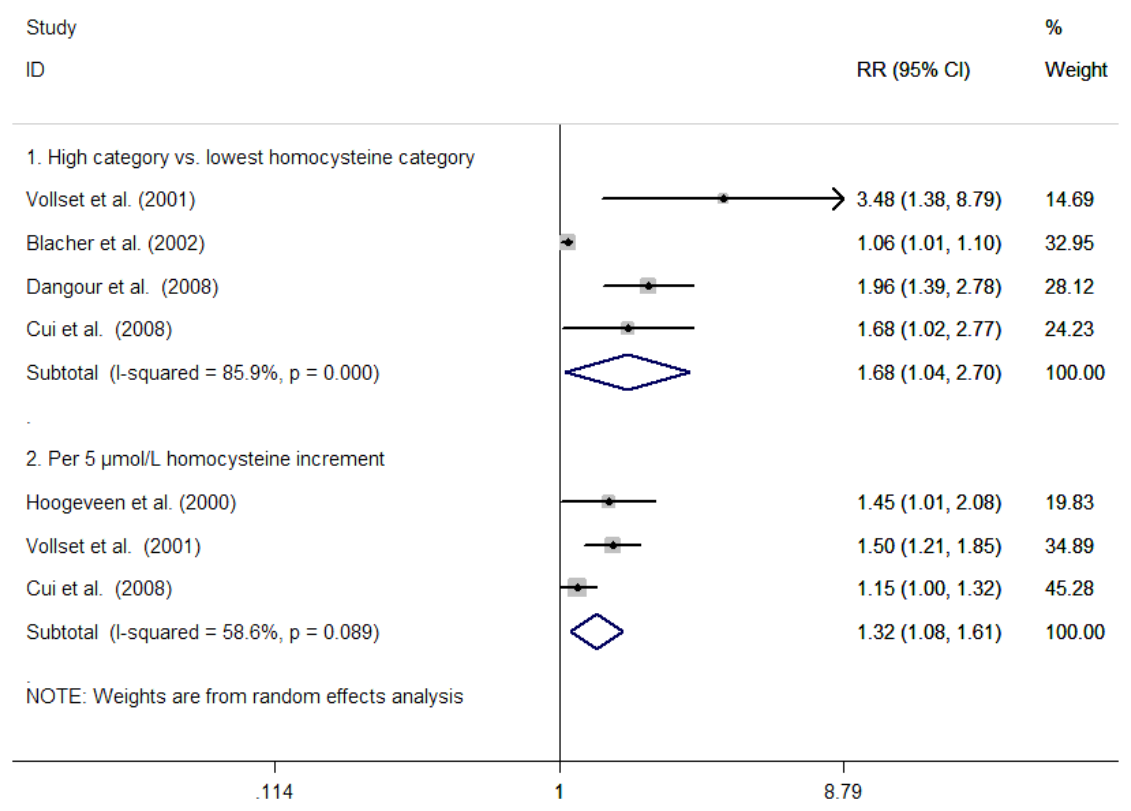

Fig. 4 Risk ratio (RR) and 95\% confidence interval (CI) from the eligible studies of homocysteine levels and cardiovascular mortality calculated separately for categorical and continuous risk estimates in a random effects model 


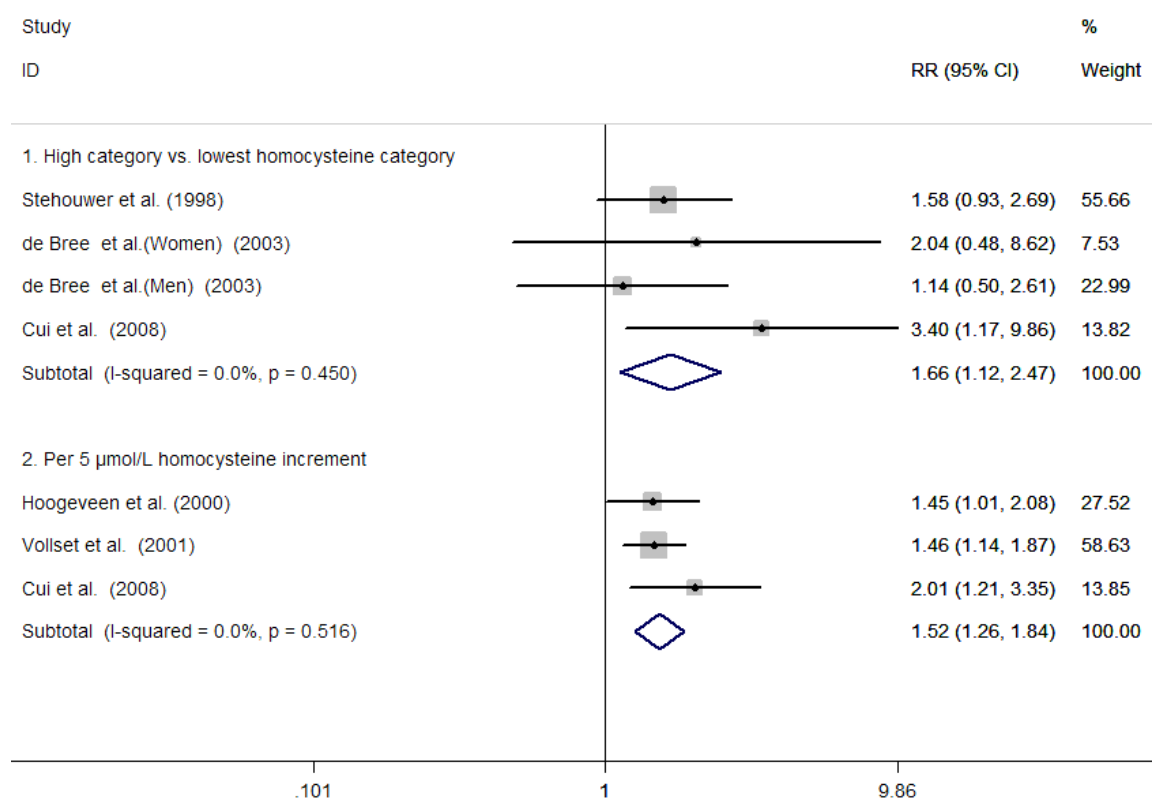

Fig. 5 Risk ratio (RR) and 95\% confidence interval (CI) from the eligible studies of homocysteine levels and coronary heart disease mortality calculated separately for categorical and continuous risk estimates in a fixed-effects model

\section{Discussion}

The findings of our current meta-analysis found that the subjects increased by $93 \%$ the risk of all-cause mortality, $68 \%$ the risk of cardiovascular mortality, and 66\% CHD mortality comparing the highest versus lowest Hcy levels. Furthermore, the dose-response analysis showed that an increment of $5 \mu \mathrm{mol} / \mathrm{L}$ Hcy increased by $27 \%$ the risk of all-cause mortality, $32 \%$ the risk of cardiovascular mortality, and $52 \%$ CHD mortality.

Total Hcy levels were higher in men than in women and increased with age (Selhub et al., 1993). Studies from the Framingham study showed that the prevalence of high Hcy was $29.3 \%$ for the entire cohort and over $40 \%$ for individuals aged 80 years and older (Selhub, 2006), although elevated Hcy levels appeared to increase the CHD mortality for categorical risk estimates. However, the sensitivity analysis only showed some trend, the risk of CHD mortality was not statistically significant (RR1.48; 95\% CI 0.97-2.27) comparing the highest to lowest Hcy. These results could be partly explained by the age of the subjects included in the studies who were relatively young, and their ages might have affected the findings of the pooled estimates. Elevated Hcy levels were not a strong risk factor for mortality in relatively young subjects (de Bree et al., 2003).

Subgroup analysis based on gender indicated that women with elevated Hcy levels increased the risk of all-cause mortality (RR 1.74; 95\% CI 1.24-2.44); in contrast, this positive association was not statistically significant among men (RR 1.87; 95\% CI 0.64-5.50). The present results suggest that the relationship between Hcy levels and all-cause mortality appears to be more pronounced in women. However, due to the limited number of studies, the results might be not robust enough to draw a conclusion. Therefore, whether men or women with elevated Hcy had more risk of mortality is still unclear.

The mechanisms underlying Hcy levels and risk of mortality have not yet been elucidated. Experimental studies suggest that higher Hcy caused endothelial dysfunction (Celermajer et al., 1993), platelet activation and thrombus formation (Dionisio et al., 2010). Meta-analysis of prospective cohort studies demonstrated that hyperhomocysteinemia was associated with cardiovascular disease (Bautista et al., 2002), cognitive decline (Ho et al., 2011), and fracture (Yang et al., 2012). All above diseases increased death in elderly persons. 
Our meta-analysis had several strengths. First, the present analysis included only prospective observational studies, which greatly reduced the selection bias. Moreover, according to the NOS, all the included studies belonged to a high quality class. Second, this meta-analysis only included multivariable adjusted OR or RR. Third, the robustness of the Hcy level as a predictor of mortality was significantly reinforced by per $5 \mu \mathrm{mol} / \mathrm{L}$ increment Hcy as a continuous variable. Several potential limitations of this study should be mentioned. First, Hcy levels are influenced by a complex interaction of environmental and genetic factors, such as nutrition, lifestyle, renal function and hormonal factors. However, the included study did not adjust some important confounding factors, such as folate and vitamin $\mathrm{B}_{12}$ levels. Additionally, there were variant adjusted confounding factors from the individual studies. Second, the ages of the subjects included in most of the studies were elderly; so the positive association between Hcy levels and death risk could not be generalized to younger persons. Third, four studies (Stehouwer et al., 1998; Kark et al., 1999; de Bree et al., 2003; Dangour et al., 2008) measured Hcy levels based on non-fasting blood samples and this might underestimate the associations with mortality due to regression dilution bias. Fourth, the number of studies was small for subgroup analysis, which might exert bias. Finally, most of the studies were from European populations, additional studies in Asian populations should be investigated to generalize our findings.

\section{Conclusions}

The findings of this meta-analysis suggest that the elevated Hcy level is an independent predictor for subsequent cardiovascular mortality or all-cause mortality in the general population, and that the risks are more pronounced among elderly persons. However, more prospective studies in different regions are needed to further investigate this association. Additionally, more well-designed randomized clinical trials are also needed to test the effects of Hcylowering therapy on the mortality events in the general population.

\section{Compliance with ethics guidelines}

Hui-yong PENG, Chang-feng MAN, Juan XU, and Yu FAN declare that they have no conflict of interest.

This article does not contain any studies with human or animal subjects performed by any of the authors.

\section{References}

Bautista, L.E., Arenas, I.A., Penuela, A., et al., 2002. Total plasma homocysteine level and risk of cardiovascular disease: a meta-analysis of prospective cohort studies. $J$. Clin. Epidemiol., 55(9):882-887. [doi:10.1016/S08954356(02)00434-1]

Begg, C.B., Mazumdar, M., 1994. Operating characteristics of a rank correlation test for publication bias. Biometrics, 50(4):1088-1101. [doi:10.2307/2533446]

Blacher, J., Benetos, A., Kirzin, J.M., et al., 2002. Relation of plasma total homocysteine to cardiovascular mortality in a French population. Am. J. Cardiol., 90(6):591-595. [doi:10.1016/S0002-9149(02)02561-4]

Bostom, A.G., Silbershatz, H., Rosenberg, I.H., et al., 1999. Nonfasting plasma total homocysteine levels and allcause and cardiovascular disease mortality in elderly Framingham men and women. Arch. Intern. Med., 159(10):1077-1080. [doi:10.1001/archinte.159.10.1077]

Celermajer, D.S., Sorensen, K., Ryalls, M., et al., 1993. Impaired endothelial function occurs in the systemic arteries of children with homozygous homocystinuria but not in their heterozygous parents. J. Am. Coll. Cardiol., 22(3):854-858. [doi:10.1016/0735-1097(93)90203-D]

Colon Lopez, V., Haan, M.N., Aiello, A.E., et al., 2008. Fasting total homocysteine (THCY) concentration and mortality in older Mexican Americans. J. Nutr. Health. Aging, 12(10):685-689.

Cui, R., Moriyama, Y., Koike, K.A., et al., 2008. Serum total homocysteine concentrations and risk of mortality from stroke and coronary heart disease in Japanese: the JACC study. Atherosclerosis, 198(2):412-418. [doi:10.1016/j. atherosclerosis.2007.09.029]

Dangour, A.D., Breeze, E., Clarke, R., et al., 2008. Plasma homocysteine, but not folate or vitamin B-12, predicts mortality in older people in the United Kingdom. J. Nutr., 138(6): 1121-1128.

de Bree, A., Verschuren, W.M., Blom, H.J., et al., 2003. Coronary heart disease mortality, plasma homocysteine, and B-vitamins: a prospective study. Atherosclerosis, 166(2):369-377. [doi:10.1016/S0021-9150(02)00373-8]

Dionisio, N., Jardin, I., Salido, G.M., et al., 2010. Homocysteine, intracellular signaling and thrombotic disorders. Curr. Med. Chem., 17(27):3109-3119. [doi:10.2174/ 092986710791959783]

Egger, M., Davey Smith, G., Schneider, M., et al., 1997. Bias in meta-analysis detected by a simple, graphical test. $B M J$, 315(7109):629-634. [doi:10.1136/bmj.315.7109.629]

Gonzalez, S., Huerta, J.M., Fernandez, S., et al., 2007. Homocysteine increases the risk of mortality in elderly individuals. Br. J. Nutr., 97(6):1138-1143. [doi:10.1017/ S0007114507691958] 
Higgins, J.P., Thompson, S.G., Deeks, J.J., et al., 2003. Measuring inconsistency in meta-analyses. $B M J$, 327(7414):557-560. [doi:10.1136/bmj.327.7414.557]

Ho, R.C., Cheung, M.W., Fu, E., et al., 2011. Is high homocysteine level a risk factor for cognitive decline in elderly? A systematic review, meta-analysis, and metaregression. Am. J. Geriatr. Psychiatry, 19(7):607-617. [doi:10.1097/JGP.0b013e3181f17eed]

Hoogeveen, E.K., Kostense, P.J., Jakobs, C., et al., 2000. Hyperhomocysteinemia increases risk of death, especially in type 2 diabetes: 5 -year follow-up of the Hoorn study. Circulation, 101(13):1506-1511. [doi:10.1161/01. CIR.101.13.1506]

Kark, J.D., Selhub, J., Adler, B., et al., 1999. Nonfasting plasma total homocysteine level and mortality in middleaged and elderly men and women in Jerusalem. Ann. Intern. Med., 131(5):321-330. [doi:10.7326/0003-4819131-5-199909070-00002]

Naess, H., Nyland, H., Idicula, T., et al., 2013. C-reactive protein and homocysteine predict long-term mortality in young ischemic stroke patients. J. Stroke. Cerebrovasc. Dis., 22(8):e435-e440. [doi:10.1016/j.jstrokecerebrovasdis. 2013.04.031]

Nygård, O., Vollset, S.E., Refsum, H., et al., 1995. Total plasma homocysteine and cardiovascular risk profile. The Hordaland homocysteine study. JAMA, 274(19):15261533. [doi:10.1001/jama.274.19.1526]

Nygård, O., Nordrehaug, J.E., Refsum, H., et al., 1997. Plasma homocysteine levels and mortality in patients with coronary artery disease. N. Engl. J. Med., 337(4):230-236 [doi:10.1056/NEJM199707243370403]

Rossi, G.P., Maiolino, G., Seccia, T.M., et al., 2006. Hyperhomocysteinemia predicts total and cardiovascular mortality in high-risk women. J. Hypertens., 24(5):851-859. [doi:10.1097/01.hjh.0000222754.75196.5c]

Selhub, J., 2006. The many facets of hyperhomocysteinemia: studies from the Framingham cohorts. J. Nutr., 136(6S): $1726 \mathrm{~S}-1730 \mathrm{~S}$.

Selhub, J., Jacques, P.F., Wilson, P.W., et al., 1993. Vitamin status and intake as primary determinants of homocysteinemia in an elderly population. JAMA, 270(22):26932698. [doi:10.1001/jama.1993.03510220049033]

Stehouwer, C.D., Weijenberg, M.P., van den Berg, M., et al., 1998. Serum homocysteine and risk of coronary heart disease and cerebrovascular disease in elderly men: a 10-year follow-up. Arterioscler. Thromb. Vasc. Biol., 18(12):1895-1901. [doi:10.1161/01.ATV.18.12.1895]

Stroup, D.F., Berlin, J.A., Morton, S.C., et al., 2000. Metaanalysis of observational studies in epidemiology: a proposal for reporting. Meta-analysis of observational studies in epidemiology (MOOSE) group. JAMA, 283(15): 2008-2012. [doi:10.1001/jama.283.15.2008]
Swart, K.M., van Schoor, N.M., Blom, H.J., et al., 2012. Homocysteine and the risk of nursing home admission and mortality in older persons. Eur. J. Clin. Nutr., 66(2): 188-195. [doi:10.1038/ejcn.2011.186]

Vollset, S.E., Refsum, H., Tverdal, A., et al., 2001. Plasma total homocysteine and cardiovascular and noncardiovascular mortality: the hordaland homocysteine study. Am. J. Clin. Nutr., 74(1):130-136.

Waśkiewicz, A., Sygnowska, E., Broda, G., 2012. Homocysteine concentration and the risk of death in the adult polish population. Kardiol. Pol., 70(9):897-902.

Wells, G., Shea, B.O., Connell, D., et al., 2013. The NewcastleOttawa scale (NOS) for assessing the quality if nonrandomized studies in meta-analyses. Available from http:// www.ohri.ca/programs/clinical_epidemiology/oxford.asp [Accessed on Oct. 6, 2013].

Wong, Y.Y., Almeida, O.P., Mccaul, K.A., et al., 2012. Homocysteine, frailty, and all-cause mortality in older men: the health in men study. J. Gerontol. A Biol. Sci. Med. Sci., 68(5):590-598. [doi:10.1093/gerona/gls211]

Yang, J., Hu, X., Zhang, Q., et al., 2012. Homocysteine level and risk of fracture: a meta-analysis and systematic review. Bone, 51(3):376-382. [doi:10.1016/j.bone.2012. 05.024]

Zylberstein, D.E., Bengtsson, C., Bjorkelund, C., et al., 2004. Serum homocysteine in relation to mortality and morbidity from coronary heart disease: a 24-year follow-up of the population study of women in Gothenburg. Circulation, 109(5):601-606. [doi:10.1161/01.CIR.0000 112581.96154.EA]

\section{中文概要}

题 目: 血浆同型半胱氨酸水平升高与心血管疾病及各种 死亡风险因子的 meta 分析

目 的: 探讨普通人群血浆同型半胱氨酸水平升高是否能 独立预测死亡风险。

创新点: 通过汇总 12 篇前瞻性研究, 定量分析血浆同型 半胱氨酸水平升高与心血管疾病及各种死亡风 险因子的关系。

方 法: 通过检索 PubMed 和 Embase 数据库, 收集普通 人群中血浆同型半胱氨酸水平与死亡关系的前 瞻性观察性研究, 采用 STATA 统计软件进行 meta 分析。

结 论: 血浆同型半胱氨酸水平升高是心血管疾病及各种 原因死亡风险增加的一个独立预测因素, 在老年 人群中尤为明显。

关键词: 同型半胱氨酸; 冠状动脉疾病; 心血管死亡; 全因性死亡; meta 分析 